\title{
SABCS: Neues zur Adjuvanz und Immuntherapie des Mammakarzinoms
}

In San Antonio wurden auch 2017 zahlreiche Studien präsentiert. Beim frühen Brustkrebs waren einige der klinisch relevanten Themen dosisdichte Chemotherapie und Dauer der erweiterten adjuvanten Hormontherapie. Neues gab es auch zur Immuntherapie bei HER2-positiven fortgeschrittenen Tumoren.

\section{Dosisdichte Chemotherapie als Goldstandard bestätigt}

Die adjuvante dosisdichte Chemotherapie bei hohem Rezidivrisiko wird seit Jahren kontrovers diskutiert. Auf dem San Antonio Breast Cancer Symposium (SABCS) präsentierten Gray et al. [1] nun eine große EBCTCG-Metaanalyse von 25 Studien mit rund 34000 Patientinnen zum Vergleich verschiedener dosisdichter Strategien (2-wöchentlich, 3- und 2-wöchentliche Sequenz) jeweils mit einer konventionellen 3-wöchentlichen Chemotherapie. Die dosisdichte und/oder sequenzielle Chemotherapie reduzierte signifikant das relative Rezidivrisiko um 15\% und die brustkrebsspezifische 10-Jahres-Mortalität um 13\%. Der Effekt war unabhängig vom Hormonrezeptor(HR)-Status und von anderen Tumor- oder Patienteneigenschaften. Somit kann eine dosisdichte Chemotherapie (mit G-CSF-Support) das Rezidivrisiko bei den meisten Hochrisikopatientinnen gut senken, bei gleichzeitig kürzerer Therapiedauer. Toxizität und therapieassoziierte Sterblichkeit waren vergleichsweise nicht erhöht.

\section{Jahre adjuvante Hormontherapie reicht aus}

Beim HR-positiven Brustkrebs tritt etwa die Hälfte der Rezidive erst nach 5 Jahren auf, weshalb bei erhöhtem Rückfallrisiko häufig nach der üblichen 5-jährigen Hormonbehandlung eine verlängerte Therapie mit Aromatasehemmern (AI) erfolgt. Das krankheitsfreie Überleben (DFS) kann damit nachweislich verbessert werden, doch die optimale Dauer der Therapieverlängerung war bisher unklar.

Die österreichische ABCSG 16/SALSA-Studie [2] zeigte jetzt, dass eine Therapieverlängerung von mehr als 2 Jahren das Therapieergebnis nicht weiter verbessert, sondern nur zu mehr Nebenwirkungen führt. In der Studie erhielten 3484 postmenopausale Frauen mit HR-positivem frühem Brustkrebs, die nach 5 Jahren Tamoxifen und/oder AI rezidivfrei waren, für weitere 2 oder 5 Jahre Anastrozol. Nach median 9 Jahren war das DFS in beiden Gruppen gleich (71,1 vs. 70,3\%; Hazard Ratio (HR): 1,007; p = $0,925)$. Allerdings nahm die Therapieadhärenz in der 5-Jahres-Gruppe stetig ab; die Frakturrate stieg in dieser Gruppe auf 6,3\% versus $4,7 \%$ in der 2-Jahres-Gruppe ( $\mathrm{p}=0,053)$. Bisher ließen sich keine Subgruppen identifizieren, die von einer längeren Therapie profitieren könnten; weitere Biomarkeranalysen folgen. Zum jetzigen Zeitpunkt erscheinen mehr als 7 Jahre einer endokrinen Therapie bei den meisten Patientinnen nicht sinnvoll.

\section{CDK4/6-Inhibition in der Prämenopause}

Ein weiteres Highlight waren die Ergebnisse der Phase-III-Studie MONALEESA-7 [3], in der erstmals ein CDK4/6-Inhibitor in einem ausschließlich prämenopausalen Kollektiv untersucht wurde. 672 prä- oder perimenopausale Patientinnen erhielten eine endokrine Erstlinientherapie (Goserelin plus Tamoxifen oder AI) plus den CDK4/6-Inhibitor Ribociclib oder Placebo. Die Zugabe von Ribociclib verdoppelte das progressionsfreie Überleben statistisch hochsignifikant (23,8 vs. 13,0 Monate; HR: 0,55; $\mathrm{p}=0,0000000983)$. Auch das objektive Ansprechen war signifikant verbessert $(50,9$ vs. $36,4 \% ; p<0,0003)$. Dieser Vorteil war in allen Subgruppen vorhanden und war unabhängig vom endokrinen Kombinationspartner. Es gab keine neuen Sicherheitssignale für Ribociclib mit Neutropenie als häufigster (61\% Grad 3/4) und in der Praxis gut beherrschbarer Nebenwirkung. Nur 2,1\% der Patientinnen hatten eine febrile Neutropenie. Im Ribociclib-Arm blieb die Lebensqualität deutlich länger erhalten, unter anderem durch eine anhaltende Schmerzverbesserung bereits nach 8 Wochen.

\section{Pembrolizumab bei Trastuzumab-Resistenz wirksam}

Nicht zuletzt konnte mit der frühen, aber dennoch bedeutsamen PhaseIb/II-Studie PANACEA/Keynote-014-Studie [4] gezeigt werden, dass zumindest ein Teil der Patientinnen mit einem fortgeschrittenen, Trastuzumab-resistenten HER2-positiven Mammakarzinom von einer Immuntherapie mit dem PD1-Antikörper Pembrolizumab profitieren könnte. Während die PD-L1-negative Gruppe ( $\mathrm{n}=12)$ nicht auf die Kombination mit Pembrolizumab plus Trastuzumab ansprach, zeigte die PD-L1-positive Gruppe ( $n=40)$ ein Ansprechen von 15\% und eine Krankheitskontrollrate von $24 \%$, unabhängig vom HR-Status. Am meisten profitierten PD-L1positive Patientinnen mit mehr als 5\% tumorinfiltrierenden Lymphozyten in den Metastasen (Ansprechen 39\%; Krankheitskontrolle 47\%). Die mediane Ansprechdauer lag bei 11,1 Monaten. Insgesamt spricht zwar nur ein relativ kleiner Anteil der Patienten auf den Checkpoint-Inhibitor an; dies scheint aber lang anhaltend zu sein. Zur Patientenselektion könnte sich hierbei neben der PD-L1-Expression auch die Lymphozyteninfiltration eignen.

Dr. med. Gerhard Emrich

\author{
Literatur \\ 1 Gray R, et al: SABCS 2017; abstr GS1-01. \\ 2 Gnant M, et al: SABCS 2017;abstr GS3-01. \\ 3 Tripathy D, et al:. SABCS 2017; abstr GS2-05. \\ 4 Loi S, et al: SABCS 2017; abstr GS2-06.
}

